\title{
Numerical Simulation and Theoretical Analysis of Perturbations in Hypersonic Boundary Layers
}

\author{
Anatoli Tumin* \\ University of Arizona, Tucson, Arizona 85721 \\ and \\ Xiaowen Wang $\ddagger$ and Xiaolin Zhong \\ University of California, Los Angeles, Los Angeles, California 90095
}

DOI: $\underline{10.2514 / 1 . J 050431}$

\begin{abstract}
Direct numerical simulations on the receptivity of hypersonic boundary layers over a flat plate and a sharp wedge were carried out with two-dimensional periodic-in-time wall blowing-suction introduced into the flow through a slot. The freestream Mach numbers were equal to 5.92 and 8 in the cases of the adiabatic flat plate and sharp wedge, respectively. The perturbation flowfield was decomposed into normal modes with the help of the multimode decomposition technique based on the spatial biorthogonal eigenfunction system. The decomposition allowed for the filtering out of stable and unstable modes hidden behind perturbations of another physical nature.
\end{abstract}

\section{Introduction}

$\mathbf{T}$ HE progress being made in computational fluid dynamics provides an opportunity for reliable simulations of such complex phenomena as laminar-turbulent transition. The dynamics of flow transition depends on the instability of small perturbations excited by external sources. Computational results provide complete information about the flowfield that would be impossible to measure in real experiments.

Recently, a method of normal mode decomposition was developed for two- and three-dimensional perturbations in compressible and incompressible boundary layers [1-3]. In [4], the method was applied to the theoretical analysis of a perturbation flowfield in the vicinity of a blowing-suction actuator obtained from direct numerical simulation (DNS). The results demonstrated very good agreement between the amplitudes of the modes filtered out from the DNS data and those solved by linear theory of the flow receptivity to wall blowing-suction. However, the development of the perturbations downstream from the actuator has not been analyzed yet.

Perturbations observed in experiments and computations in the vicinity of an actuator possess a nonmonotonic character. This behavior occurs because the perturbation introduced by the actuator is composed of modes of the discrete (unstable and stable modes) and continuous spectra, and one cannot distinguish the unstable mode clearly. Are the observations still compatible with the linear stability theory (LST)? To answer this question, we must decompose the perturbation into the normal modes and compare their amplitudes with those predicted by LST. However, the LST prediction must take into account the nonparallel boundary-layer flow effects, because the development of the perturbation takes place on a length scale much larger than the boundary-layer thickness.

The nonparallel flow effects on the development of unstable discrete modes on a length scale that is much larger than the

Presented as Paper 2010-0534 at the 48th AIAA Aerospace Sciences Meeting, Orlando, FL, 4-7 January 2010; received 12 January 2010; revision received 26 August 2010; accepted for publication 26 October 2010. Copyright (C 2010 by the American Institute of Aeronautics and Astronautics, Inc. All rights reserved. Copies of this paper may be made for personal or internal use, on condition that the copier pay the $\$ 10.00$ per-copy fee to the Copyright Clearance Center, Inc., 222 Rosewood Drive, Danvers, MA 01923; include the code 0001-1452/11 and \$10.00 in correspondence with the CCC.

*Professor, Department of Aerospace and Mechanical Engineering, Associate Fellow AIAA.

${ }^{\dagger}$ Research Associate, Mechanical and Aerospace Engineering Department, Member AIAA.

†Professor, Mechanical and Aerospace Engineering Department, Associate Fellow AIAA. boundary-layer thickness have been studied within the scope of the method of multiple scales (MMS) [5-13]. Another method that allows inclusion of the nonparallel flow effects on unstable modes is based on the parabolized stability equations [14-16]. Fedorov and Khokhlov [17] pointed out that the role of decaying modes can be significant, and one must pay attention to them if there is a synchronism with the other modes. Because the analysis of decaying modes is important, one must include the nonparallel boundary-layer flow effects using the MMS.

In the present work, we apply the multimode decomposition to DNS results downstream from the blowing-suction actuator in hypersonic boundary layers past a flat plate and a sharp wedge to compare the amplitudes of the modes found from the computations with the prediction of the LST including nonparallel flow effects.

\section{Outline of Multimode Decomposition}

The method of multimode decomposition of perturbations having a prescribed frequency is based on the biorthogonal eigenfunction system for linearized Navier-Stokes equations [3]. For the clarity of further discussion, we reproduce the main definitions necessary for discussing the present work.

We consider a compressible two-dimensional boundary layer in Cartesian coordinates, where $x$ and $z$ are the downstream and spanwise coordinates, respectively, and coordinate $y$ corresponds to the distance from the wall. We write the linearized Navier-Stokes equations for a periodic-in-time perturbation (the frequency is equal to zero in the case of a roughness-induced perturbation), $\sim \exp (-i \omega t)$, in matrix form as

$$
\frac{\partial}{\partial y}\left(\mathbf{L}_{0} \frac{\partial \mathbf{A}}{\partial y}\right)+\mathbf{L}_{1} \frac{\partial \mathbf{A}}{\partial y}=\mathbf{H}_{1} \mathbf{A}+\mathbf{H}_{2} \frac{\partial \mathbf{A}}{\partial x}+\mathbf{H}_{3} \frac{\partial \mathbf{A}}{\partial z}+\mathbf{H}_{4} \mathbf{A}
$$

where vector $\mathbf{A}$ has 16 components

$$
\begin{gathered}
\mathbf{A}(x, y, z)=(u, \partial u / \partial y, v, \pi, \theta, \partial \theta / \partial y, w, \partial w / \partial y, \partial u / \partial x, \\
\partial v / \partial x, \partial \theta / \partial x, \partial w / \partial x, \partial u / \partial z, \partial v / \partial z, \partial \theta / \partial z, \partial w / \partial z)^{T}
\end{gathered}
$$

$\mathbf{L}_{0}, \mathbf{L}_{1}, \mathbf{H}_{1}, \mathbf{H}_{2}, \mathbf{H}_{3}$, and $\mathbf{H}_{4}$ are $16 \times 16$ matrices (their definitions are given in [18]); $u, v, w, \pi$, and $\theta$ represent three velocity components, pressure, and temperature perturbations, respectively; and the superscript $T$ in Eq. (2) stands for transpose. Matrix $\mathbf{H}_{4}$ originates from the nonparallel character of the flow. It includes terms with the $y$ component of the mean flow velocity and derivatives of the mean flow profiles with respect to the coordinate $x$.

In the quasi-parallel flow approximation, the solution of the linearized Navier-Stokes equations can be expanded into normal 
modes of the discrete and continuous spectra $\left\{\mathbf{A}_{\alpha \beta}, \mathbf{B}_{\alpha \beta}\right\}$ [3] , where $\mathbf{A}_{\alpha \beta}$ and $\mathbf{B}_{\alpha \beta}$ are eigenfunctions of the direct and adjoint problems. Subscripts $\alpha$ and $\beta$ indicate the eigenfunctions corresponding to the streamwise $\alpha$ and spanwise $\beta$ wave numbers, respectively. The eigenfunction system $\left\{\mathbf{A}_{\alpha \beta}, \mathbf{B}_{\alpha \beta}\right\}$ has an orthogonality relation given as

$$
\left\langle\mathbf{H}_{2} \mathbf{A}_{\alpha \beta}, \mathbf{B}_{\alpha^{\prime} \beta}\right\rangle \equiv \int_{0}^{\infty}\left(\mathbf{H}_{2} \mathbf{A}_{\alpha \beta}, \mathbf{B}_{\alpha^{\prime} \beta}\right) \mathrm{d} y=\Gamma \Delta_{\alpha \alpha^{\prime}}
$$

where $\Gamma$ is a normalization constant, $\Delta_{\alpha \alpha^{\prime}}$ is a Kronecker delta if either $\alpha$ or $\alpha^{\prime}$ belongs to the discrete spectrum, and $\Delta_{\alpha \alpha^{\prime}}$ is a Dirac delta function if both $\alpha$ and $\alpha^{\prime}$ belong to the continuous spectrum.

The orthogonality relation in Eq. (3) can be used for decomposition of the DNS data at $x=x_{0}$ into normal modes. After applying the Fourier transform in $z$, the data can be presented in the form of a vector:

$$
\mathbf{A}_{\beta 0}\left(x_{0}, y\right)=\sum_{\alpha} C_{\alpha}(\beta) \mathbf{A}_{\alpha \beta}\left(x_{0}, y\right)
$$

where $\sum$ stands for summation and integration over the discrete and continuous spectra, respectively. Applying the orthogonality relation in Eq. (3), one can find the coefficients $C_{\alpha}(\beta)$ as follows:

$$
C_{\alpha}(\beta)=\frac{\left\langle\mathbf{H}_{\mathbf{2}} \mathbf{A}_{\beta \mathbf{0}}\left(\mathbf{x}_{\mathbf{0}}, \mathbf{y}\right), \mathbf{B}_{\alpha, \beta}\right\rangle}{\Gamma}
$$

In the case of the transient growth phenomenon studied in experiments [19-23] and DNS [24-26], the flow perturbation is given only by modes of the continuous spectrum. The multimode decomposition has been applied by Denissen and White [27] to the analysis of the DNS data of Rizzetta and Visbal [25]. The authors demonstrated that the method can be used even when only partial information in vector $\mathbf{A}_{\beta 0}\left(x_{0}, y\right)$ is available.

In a weakly nonparallel flow, one can employ the MMS by introducing fast $(x)$ and slow $(X=\varepsilon x$ and $\varepsilon \ll 1)$ scales. The mean flow profiles depend on $y$ and $X$ only, whereas the perturbation will depend on both length scales. In the case of a discrete mode, the solution of the linearized Navier-Stokes equation is presented in the form

$$
\begin{aligned}
& \mathbf{A}_{\beta}(x, X, y)=\left[D_{\nu}(X) \mathbf{A}_{\alpha_{\nu} \beta}^{(0)}(X, y) e^{i \int \alpha_{v}(X) \mathrm{d} x}\right. \\
& \left.\quad+\varepsilon \mathbf{A}_{\alpha_{v} \beta}^{(1)}(X, y) e^{i \int \alpha_{\nu}(X) \mathrm{d} x}+\cdots\right]
\end{aligned}
$$

where the function $D_{v}(X)$ must be determined. After substitution of Eq. (6) into Eq. (1), we arrive in order $O(\varepsilon)$ at an inhomogeneous equation for $\mathbf{A}_{\alpha_{\nu} \beta}^{(1)}$. The solvability condition of this equation allows for the finding of $D_{v}(X)$ (details and relevant references can be found in [18]).

\section{Direct Numerical Simulation Approach}

In DNS, the receptivity of hypersonic boundary layers over a flat plate and a sharp wedge to wall blowing-suction are considered by solving the two-dimensional compressible Navier-Stokes equations. Wall blowing-suction is introduced by a slot located near the leading edge. In the assumption of thermally and calorically perfect gas flows, the governing equations in conservative variables are given as

$$
\frac{\partial \mathbf{U}}{\partial t}+\frac{\partial}{\partial x}\left(\mathbf{F}_{1 i}+\mathbf{F}_{1 v}\right)+\frac{\partial}{\partial y}\left(\mathbf{F}_{2 i}+\mathbf{F}_{2 v}\right)=0
$$

where $\mathbf{U}$ is a column vector containing the conservative variables

$$
\mathbf{U}=\{\rho, \rho u, \rho v, e\}^{T}
$$

The flux vectors in Eq. (7) are divided into their inviscid and viscous components due to the fact that the two components are discretized with different schemes. The components $\mathbf{F}_{1 i}$ and $\mathbf{F}_{2 i}$ are inviscid flux components, whereas $\mathbf{F}_{1 v}$ and $\mathbf{F}_{2 v}$ are viscous flux components:

$$
\begin{gathered}
\mathbf{F}_{j i}=\left[\begin{array}{c}
\rho u_{j} \\
\rho u u_{j}+p \Delta_{1 j} \\
\rho v u_{j}+p \Delta_{2 j} \\
u_{j}(e+p)
\end{array}\right] \\
\mathbf{F}_{j v}=\left[\begin{array}{c}
0 \\
-\tau_{x x_{j}} \\
-\tau_{y x_{j}} \\
-\tau_{x_{k} x_{j}} u_{k}-K \frac{\partial T}{\partial x_{j}}
\end{array}\right]
\end{gathered}
$$

with $j, k \in(1,2)$. In Cartesian coordinates, $x_{1}$ and $u_{1}$ are defined in the streamwise direction ( $x$ and $u$ ), whereas $x_{2}$ and $u_{2}$ are defined in the wall-normal direction ( $y$ and $v$ ). by

Under the perfect gas assumption, pressure and energy are given

$$
p=\rho R T
$$

$$
e=\rho c_{v} T+\frac{\rho}{2}\left(u^{2}+v^{2}\right)
$$

where $c_{v}$ is the specific heat at constant volume. In the simulation, the viscosity coefficient $\mu$ and the heat conductivity $K$ are calculated using Sutherland's law together with a constant Prandtl number $P r$. They are both functions of temperature only:

$$
\begin{gathered}
\mu=\mu_{r}\left(\frac{T}{T_{r}}\right)^{3 / 2} \frac{T_{r}+T_{s}}{T+T_{s}} \\
K=\frac{\mu c_{p}}{P r}
\end{gathered}
$$

where $\mu_{r}=1.7894 \times 10^{-5} \mathrm{Ns} / \mathrm{m}^{2}, T_{r}=288.0 \mathrm{~K}, T_{s}=110.33 \mathrm{~K}$, and $c_{p}$ is the specific heat at constant pressure.

The scales of velocity and length are the freestream velocity $U_{\infty}$ and the Blasius length scale, $L=\left(\mu_{\infty} x / \rho_{\infty} U_{\infty}\right)^{1 / 2}$. Since the length scale is changing along the flat plate, it is not convenient to scale the frequency in terms of $L / U_{\infty}$. Instead, the frequency is scaled as follows:

$$
F=\frac{2 \pi f \mu_{\infty}}{\rho_{\infty} U_{\infty}^{2}}=\frac{\omega \mu_{\infty}}{\rho_{\infty} U_{\infty}^{2}}
$$

In boundary-layer analysis, the Reynolds number based on the Blasius length scale is generally used:

$$
R=\frac{\rho_{\infty} U_{\infty} L}{\mu_{\infty}}
$$

In the present work, the fifth-order shock-fitting finite difference method of Zhong [28] is used to solve the governing equations in a domain bounded by the bow shock and the flat plate (or wedge). In other words, the bow shock is treated as a boundary of the computational domain. The Rankine-Hugoniot relations across the shock and a characteristic compatibility relation coming from the downstream flowfield are combined to solve for the flow variables behind the shock. The shock-fitting method makes it possible for the Navier-Stokes equations to be spatially discretized by high-order finite difference methods. Specifically, a fifth-order upwind scheme is applied to discretize the inviscid flux derivatives. By using the shock-fitting method, the interaction between the bow shock and the wall blowing-suction induced perturbations is solved as a part of the solutions, with the position and velocity of the shock front being solved as dependent variables. Both of the cases correspond to an adiabatic wall boundary condition.

The numerical simulations in the current paper have been well validated. For the flow over the flat plate, three sets of grid structures are used to check the grid independence of numerical simulation results near the leading edge. The steady flow is compared with the experimental measurements of Maslov et al. [29]. At three different 
locations, $x=96,121$, and $138 \mathrm{~mm}(R=1134.46,1254.19$, and 1329.66); the distributions of the dimensionless streamwise velocity and normalized Mach number in the wall-normal direction are in good agreement with those measured in experiments. Two sets of the grid structures are used to check grid independence of unsteady numerical simulations. The comparison of pressure perturbation amplitudes calculated using the two grid structures shows that the grid structure used for the simulation is sufficient. Details of the validations are presented in [30]. In [4], validations of the numerical simulation results on the sharp wedge were carried out for both steady and unsteady flows. Furthermore, the numerical perturbation field just downstream of the blowing-suction slot was decomposed into boundary-layer waves. The amplitudes of the decomposed waves were in good agreement with those obtained from a theoretical receptivity model.

\section{Results}

\section{A. Flat Plate}

The freestream flow conditions that we consider are Mach number $M_{\infty}=5.92$, temperature $T_{\infty}=48.69 \mathrm{~K}$, and pressure $p_{\infty}=$ $742.76 \mathrm{~Pa}$. The Prandtl number and the specific heat ratio are 0.72 and 1.4, respectively. The freestream flow parameters are the same as those of Maslov et al. [29].

The viscosity coefficient is calculated by Sutherland's law. The dimensionless blowing-suction of mass flux at the wall is expressed as

$(\rho v)^{\prime}=\epsilon g(l) S(t), \quad \epsilon=0.405 \times 10^{-5}$,

$g(l)$

$$
= \begin{cases}20.25 l^{5}-35.4375 l^{4}+15.1875 l^{2}, & (l \leq 1) ; \\ -20.25(2-l)^{5}+35.4375(2-l)^{4}-15.1875(2-l)^{2}, & (l>1),\end{cases}
$$

$l(x)=\frac{2\left(x-x_{i}\right)}{\left(x_{e}-x_{i}\right)}, \quad x_{i} \leq x \leq x_{e}$

where $x_{i}=33 \mathrm{~mm}$ and $x_{e}=37 \mathrm{~mm}$ are the coordinates of the leading and the trailing edges of the slot, respectively. The amplitude distribution $g(l)$ is shown in Fig. 1 .

The function of time $S(t)$ in Eq. (17) is defined as

$$
S(t)= \begin{cases}1, & \bmod (t, 20 \mu \mathrm{s}) \leq 2 \mu \mathrm{s} \\ 0, & \bmod (t, 20 \mu \mathrm{s})>2 \mu \mathrm{s}\end{cases}
$$

The function $S(t)$ can be expressed as a Fourier series.

Analyses of the mean flow velocity, the temperature profiles, and their derivatives have shown that they agree well with the self-similar solution for a boundary layer over a flat plate (see Appendix A). Only the second derivatives of the velocity and temperature profiles demonstrate some differences between the DNS results and the selfsimilar solution. Because of the viscous-inviscid interaction, the

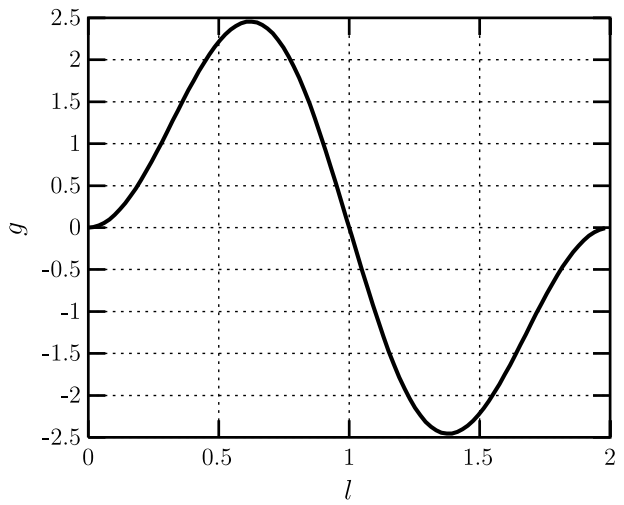

Fig. 1 Amplitude distribution of the blowing-suction through the slot.

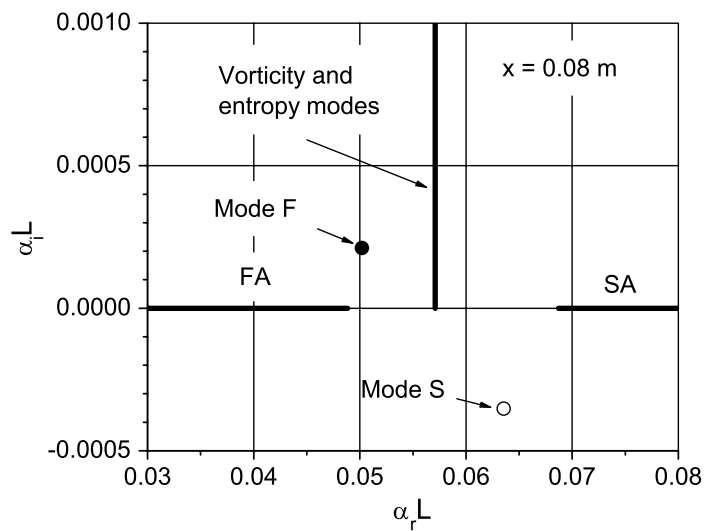

Fig. 2 Discrete modes and the continuous spectrum (FA and SA stand for fast and slow acoustic modes, respectively).

edge velocity $U_{e}$ and temperature $T_{e}$ are slightly different from the freestream values $U_{\infty}$ and $T_{\infty}$, respectively. Therefore, the local edge Mach number $M_{e}$ is also different from the freestream Mach number. However, the viscous-inviscid interaction is weak at the considered flow parameters. For example, we consider the flow parameters outside the boundary layer at $y / L \approx 50$, where the length scale $L$ is defined as $\left(\mu_{\infty} x / \rho_{\infty} U_{\infty}\right)^{1 / 2}$. At $x=0.099,0.359$, and $0.659 \mathrm{~m}$, the dimensionless velocities and the local Mach numbers are equal to $U / U_{\infty}=0.9983,0.9992$, and 0.9994, and $M_{e}=5.845,5.882$, and 5.892 , respectively. Therefore, we neglect these small variations in the local Mach number $M_{e}$, in the edge velocity $U_{e}$, and the temperature $T_{e}$, and we consider them to be equal to the freestream parameters.

Comparison of the eigenvalues $\alpha$ obtained using the self-similar and DNS profiles is shown in Appendix B. There is a difference in $\alpha_{i}$ at high frequencies. In the analysis of the flat plate data, the selfsimilar profiles have been used in the stability equations. The analysis of the perturbations is limited to the DNS data corresponding to perturbations of $100 \mathrm{kHz}$ only. The corresponding dimensionless frequency is $55.02939 \times 10^{-6}$. To deal with the twodimensional perturbations within the solver of $[\underline{3}, 18]$, the spanwise wave number $\beta$ scaled with the Blasius length scale $L$ was chosen equal to $10^{-5}$.

To illustrate further analysis of DNS results, features of the spectrum should be introduced. Figure 2 shows the branches of the continuous spectrum and two discrete modes at $x=0.08 \mathrm{~m}$ ( $R=1063.02)$. One of the discrete modes is labeled mode $F$ (fast); the other is labeled mode $S$ (slow). The mode names stem from their phase velocity features in the vicinity of the leading edge. One can see in Fig. 3 that mode $S$ is synchronized with the slow acoustic wave $\left(c_{r}=1-\overline{1} / M_{\infty}\right)$, whereas mode $F$ is synchronized with the fast acoustic wave $\left(c_{r}=1+1 / M_{\infty}\right)$. At the chosen flow parameters, mode $F$ is always stable, and mode $S$ is the unstable mode. One can see that mode $F$ is synchronized with the vorticity/entropy modes

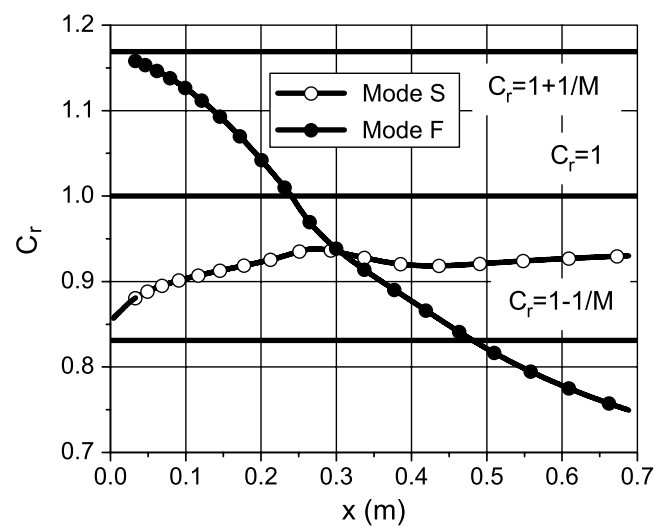

Fig. 3 Real parts of the phase velocities of the discrete modes $F$ and $S$ scaled with the freestream velocity $U_{\infty}$. 


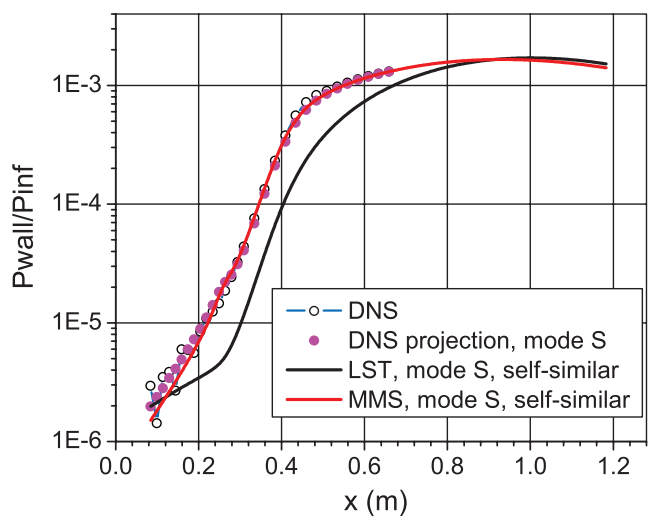

Fig. 4 Projection of the DNS results onto the discrete mode $S$.

having dimensionless phase velocity $c_{r}=1$ at $x \approx 0.25 \mathrm{~m}(R=$ 1879.17). The significance of the decaying mode $F$ stems from its synchronization with mode $S$, where the decaying mode can give rise to the unstable mode (switching of the modes), which may lead to transition [17].

\section{Mode $S$}

Figure 4 shows the pressure perturbation on the wall (scaled with the freestream pressure) obtained in the DNS and projections onto the discrete mode $S$. Amplification of the discrete mode evaluated with and without the nonparallel flow effects (MMS and LST, respectively) is also presented in Fig. 4. One can see that the nonparallel flow effect is significant in this example. The DNS data for the wall pressure perturbation have wiggles (rapid oscillations in the pressure perturbation) near the actuator region due to input from the various modes presented in the signal (Fig. 5). The filtered-out amplitude of the unstable mode $S$ is smooth, and it is in good agreement with the theoretical prediction on the whole interval.

\section{Mode F}

It is interesting to look at the filtered-out decaying mode $F$ on Fig. 6. It is in good agreement with the theoretical prediction up to $x \approx \overline{0} .25 \mathrm{~m}$. After that, it experiences a jump, and the amplitude becomes comparable with the amplitude of mode $S$. The result can be attributed to the next term in the expansion in Eq. (6). The second term, $\mathbf{A}_{\alpha_{v} \beta}^{(1)}(X, y)$, can be expanded into the eigenfunction system. It is the standard problem of finding eigenfunctions of a perturbed operator using the unperturbed basis [31]. For the nonresonance case when eigenvalues of modes $F$ and $S$ are distinct $\left(\alpha_{S} \neq \alpha_{F}\right)$, it is straightforward to find a projection of $\mathbf{A}_{\alpha_{S} \beta}^{(1)}(X, y)$ onto $\mathbf{A}_{\alpha_{F} \beta}(X, y)$ (indices $S$ and $F$ indicate slow and fast discrete modes, respectively).

After applying of the Fourier transform to the linearized Eq. (1), with respect to coordinate $z$ and substitution of $\mathbf{A}_{\beta}(x, X, y)$, one can derive the following equation for $\mathbf{A}_{\alpha_{S} \beta}^{(1)}(X, y)$ :

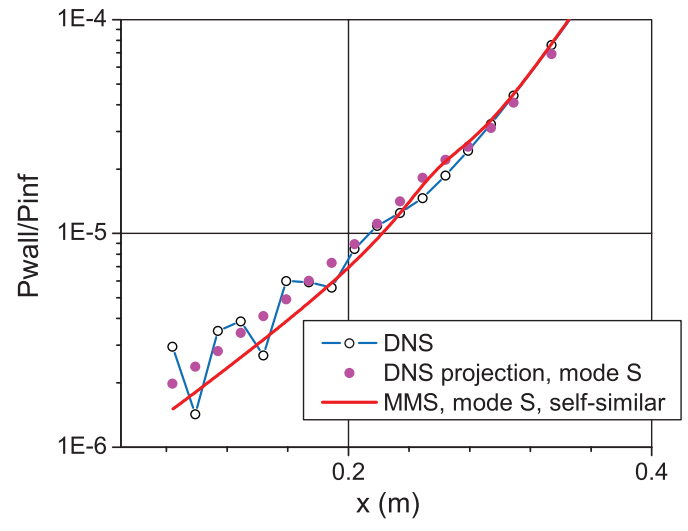

Fig. 5 A closer view of the results in Fig. $\underline{4}$ in the vicinity of the actuator.

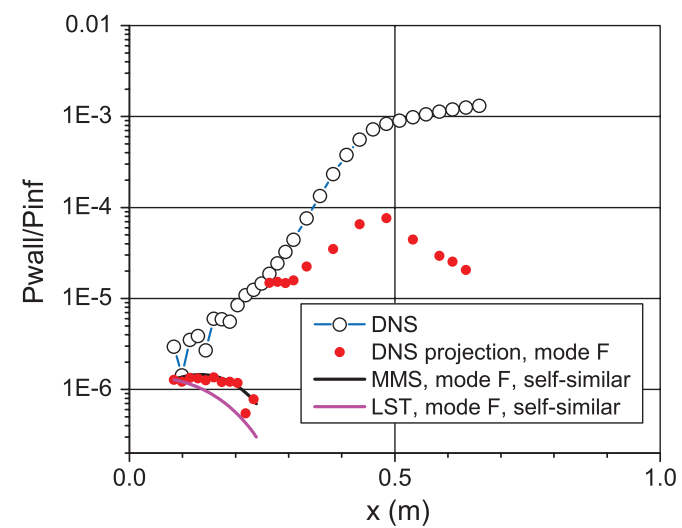

Fig. 6 Projection of the DNS results onto the discrete mode $F$.

$$
\begin{aligned}
\frac{\partial}{\partial y} & \left(\mathbf{L}_{0} \frac{\partial \mathbf{A}_{S}^{(1)}}{\partial y}\right)+\mathbf{L}_{1} \frac{\partial \mathbf{A}_{S}^{(1)}}{\partial y}-\mathbf{H}_{1} \mathbf{A}_{S}^{(1)}-i \alpha_{S} \mathbf{H}_{2} \mathbf{A}_{S}^{(1)} \\
& -i \beta \mathbf{H}_{3} \mathbf{A}_{S}^{(1)}=\boldsymbol{\Phi}, \\
\boldsymbol{\Phi} & \equiv \frac{\mathrm{d} D_{S}(X)}{\mathrm{d} X} \mathbf{H}_{2} \mathbf{A}_{S}^{(0)}+D_{S}(X) \mathbf{H}_{2} \frac{\partial \mathbf{A}_{S}^{(0)}}{\partial X}+D_{S}(X) \overline{\mathbf{H}}_{4} \mathbf{A}_{S}^{(0)}
\end{aligned}
$$

where $\overline{\mathbf{H}}_{4}=\varepsilon^{-1} \mathbf{H}_{4}$. For the purpose of brevity, we use subscript $S$ to indicate the slow discrete mode having wave numbers $\alpha_{S}$. One can represent the solution for $\mathbf{A}_{S}^{(1)}$ as an expansion into the eigenfunctions of the undisturbed operator. In symbolic form, we write

$$
\mathbf{A}_{S}^{(1)}=\sum_{\alpha_{k} \neq \alpha_{S}} C_{k}(X) \mathbf{A}_{k}^{(0)}
$$

The symbolic form of the expansion in Eq. (20) means that we include expansion into the discrete modes and continuous spectrum as well. Assuming that there is no resonance $\left(\alpha_{k} \neq \alpha_{S}\right)$, one can substitute $\mathbf{A}_{S}^{(1)}$ from Eq. (20) into Eq. (19). Using the dot product with the adjoint eigenvector $\overline{\mathbf{B}_{F}^{(0)}}$, we arrive at the coefficient $C_{F}$ :

$$
C_{F}(X)=\frac{D_{S}(X)}{i\left(\alpha_{F}-\alpha_{S}\right)} \frac{\left\langle\mathbf{H}_{2} \frac{\partial \mathbf{A}_{S}^{(0)}}{\partial X}, \mathbf{B}_{F}^{(0)}\right\rangle+\left\langle\overline{\mathbf{H}}_{4} \mathbf{A}_{S}^{(0)}, \mathbf{B}_{F}^{(0)}\right\rangle}{\left\langle\mathbf{H}_{2} \mathbf{A}_{F}^{(0)}, \mathbf{B}_{F}^{(0)}\right\rangle}
$$

The input of mode $F$ into the second term of Eq. (6) has a wave number (and phase speed) corresponding to mode $S$. We refer to this contribution of mode $F$ as $\mathrm{S} 2 \mathrm{~F}$ centaur in order to emphasize the twofold character of the term. The wall pressure perturbation associated with S2F centaur is shown in Fig. 7. Although the theoretical result for mode $F$ downstream from the point of synchronism demonstrates the same qualitative behavior as the amplitude of the

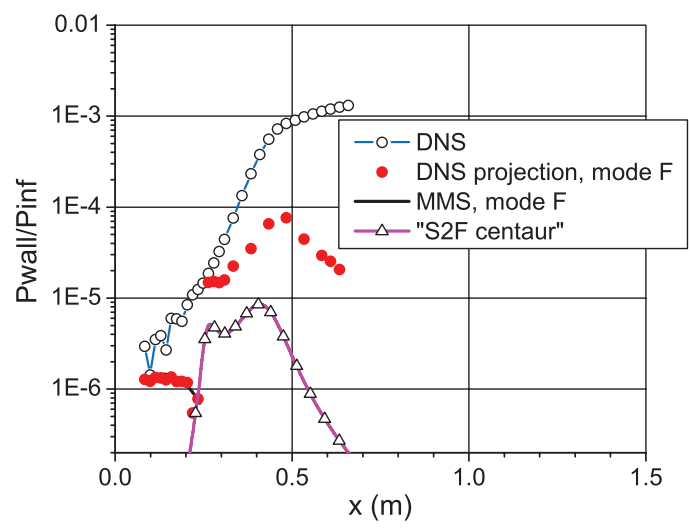

Fig. 7 Projection of the DNS results onto the discrete mode $F$ and amplitude of $\mathrm{S} 2 \mathrm{~F}$ centaur. 


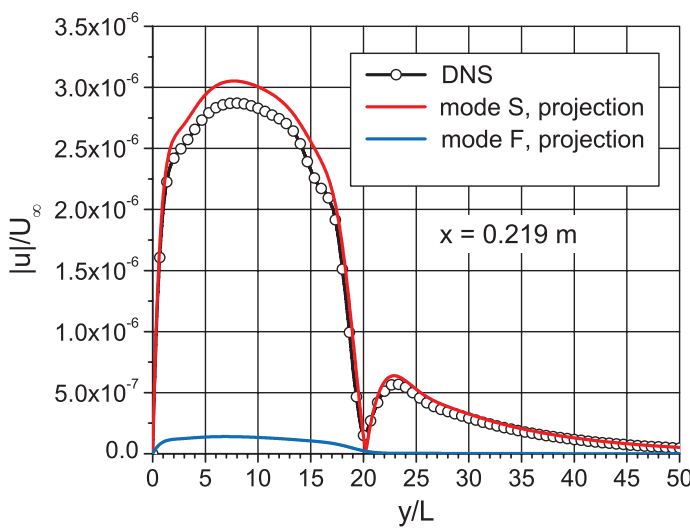

Fig. 8 Streamwise velocity perturbation at $x=0.219 \mathrm{~m}$.

DNS projection onto mode $F$, there is a quantitative discrepancy that has yet to be understood.

\section{Velocity Profiles of Modes $S$ and $F$}

Having found the coefficients in the projection of the DNS results onto modes $S$ and $F$, we can compare the velocity profiles of the modes with the DNS results in order to evaluate their significance at different distances from the actuator (see Figs. $\underline{8}-10$ ).

One can see that the main input into the velocity perturbation stems from mode $S$. In the vicinity of the modes' synchronism, the amplitude of mode $F$ is higher, as expected from results presented in Fig. 7. The receptivity studies in [4] showed that the amplitude of mode $F$ in the vicinity of the actuator is higher than the amplitude of mode $S$. In the present work, we consider perturbations far

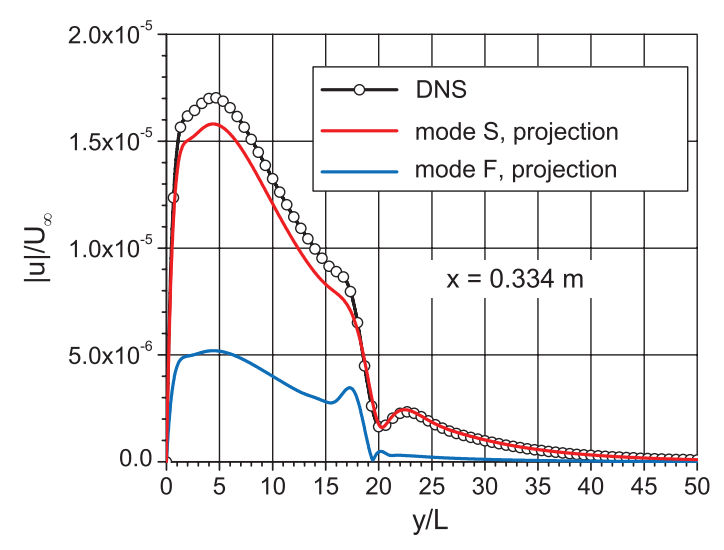

Fig. 9 Streamwise velocity perturbation at $x=0.334 \mathrm{~m}$.

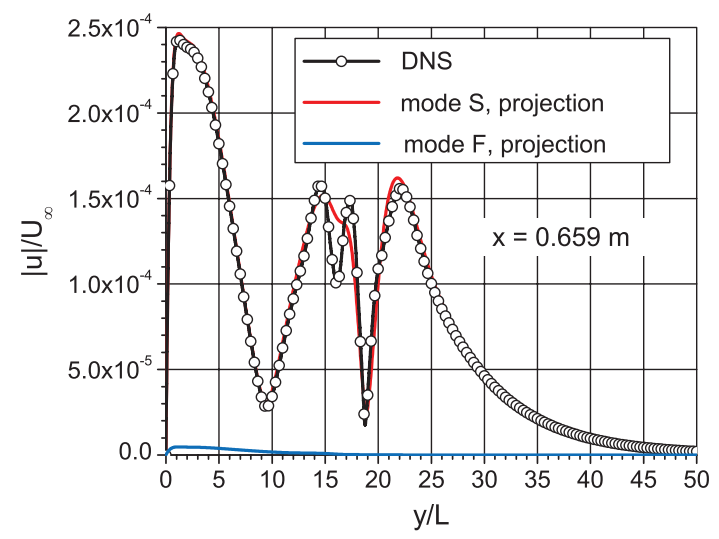

Fig. 10 Streamwise velocity perturbation at $x=0.659 \mathrm{~m}$. downstream from the actuator, and mode $S$ has a larger amplitude than mode $F$.

\section{B. Sharp Wedge}

In this example, periodic-in-time wall blowing-suction was introduced into the boundary layer over a wedge of a half-angle of $5.3 \mathrm{deg}$. The freestream flow conditions were Mach number $M_{\infty}=8$, temperature $T_{\infty}=54.8 \mathrm{~K}$, and pressure $p_{\infty}=389 \mathrm{~Pa}$. The Prandtl number and the specific heats ratio were 0.72 and 1.4 , respectively. The viscosity coefficient was calculated by Sutherland's law. The flow parameters were the same as in the work by Malik et al. [32]. Results of [32] were used for the validation of the code used in the present work (see [4]).

The periodic-in-time blowing-suction slot has coordinates of the leading and trailing edges at $x_{i}=51.84 \mathrm{~mm}$ and $x_{e}=63.84 \mathrm{~mm}$, respectively. These flow parameters and the actuator location correspond to case 3 considered in [4]. The dimensionless wall blowing-suction of mass flux at the wall is expressed as

$$
(\rho v)^{\prime}=q_{0} g(l) \sum_{n=1}^{15} \sin \left(\omega_{n} t\right)
$$

where $q_{0}=0.734 \times 10^{-7}$ is a dimensionless amplitude parameter, scaled by the freestream streamwise mass flux; function $g(l)$ is defined in Eq. (17), and $\omega_{n}$ is the circular frequency of the multifrequency perturbations.

Figure 11 illustrates the pressure perturbations on the wedge at three frequencies: $44.76,104.44$, and $164.12 \mathrm{kHz}$ (the dimensionless frequencies $\omega \mu_{e} /\left(\rho_{e} U_{e}^{2}\right)$ scaled with the local boundary-layer edge parameters are approximately $21.4 \times 10^{-6}, 50.0 \times 10^{-6}$, and $78.6 \times 10^{-6}$, respectively). Figure 12 shows the local Reynolds number $R=\left(\rho_{e} x / \mu_{e} U_{e}\right)^{1 / 2}$ versus coordinate $x$.

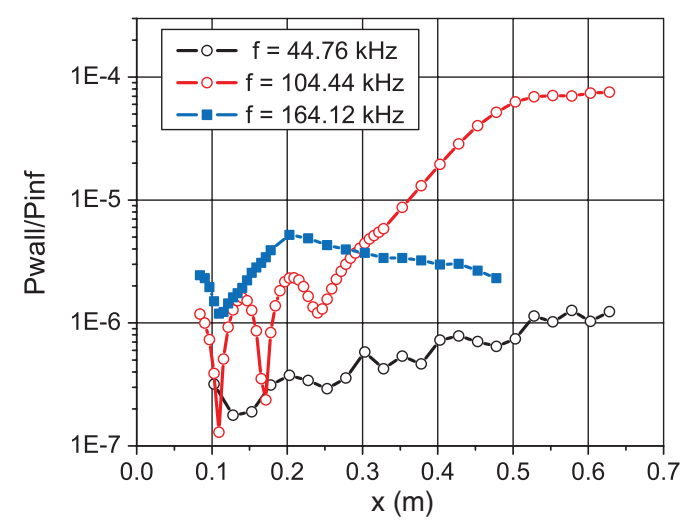

Fig. 11 Pressure perturbations $\left(p_{\text {wall }} / p_{\infty}\right)$ on the wedge at three frequencies.

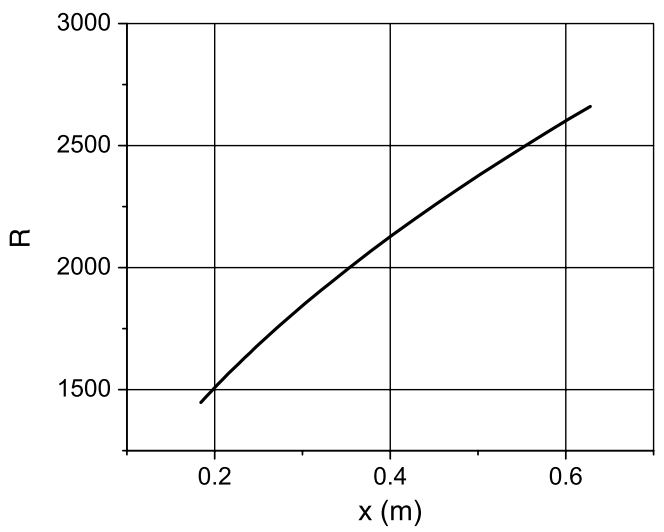

Fig. 12 The local Reynolds number versus coordinate $x$. 


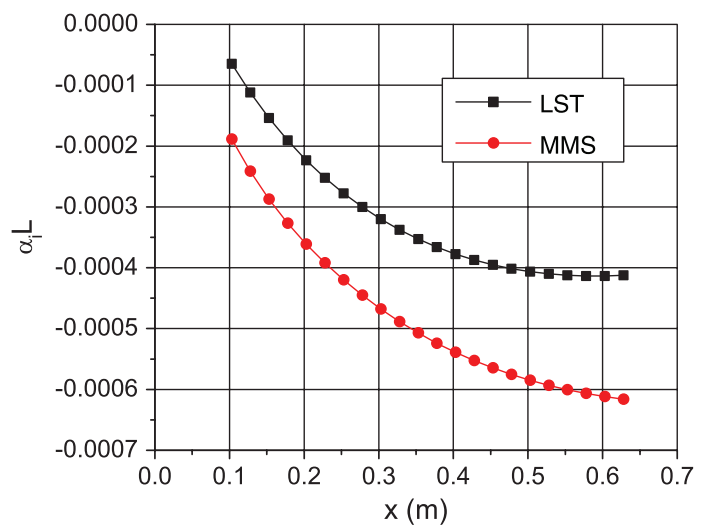

Fig. $13 \operatorname{Im}\left(\alpha_{i}\right)$ versus $x$ at frequency $f=44.76 \mathrm{kHz}$.

In the following examples, analysis of the flow stability was based on the velocity and temperature profiles obtained from the computations without an assumption about the self-similar character of the boundary-layer flow. To compare the projected amplitudes with those predicted using the MMS, we need derivatives $\partial / \partial x$ of the streamwise velocity and temperature profiles of the mean flow. These derivatives were derived using the computational profiles together with the assumption that the profiles locally are self-similar.

Figure 13 shows the imaginary part of the wave number $\alpha$ scaled with $L=\left(\mu_{\infty} x / \rho_{\infty} U_{\infty}\right)^{1 / 2}$, obtained using the quasi-parallel approximation (LST) and using the MMS for perturbations of $F=44.6 \mathrm{kHz}$. Figure 14 shows wall pressure perturbations in DNS results, and in their projection onto mode $S$, together with the

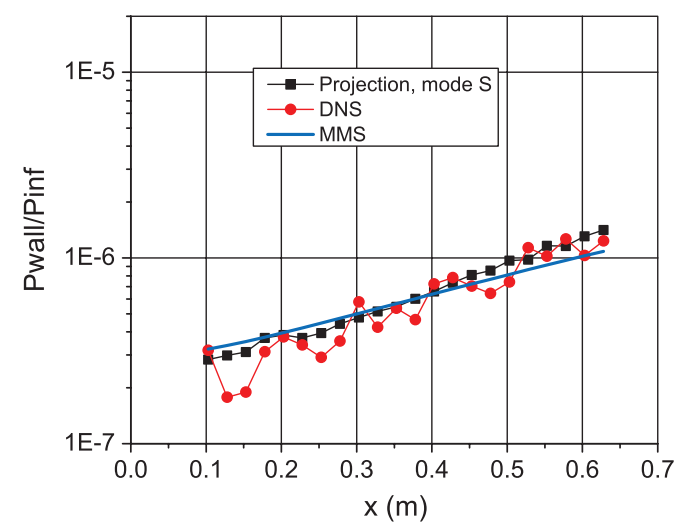

Fig. 14 Projection of the DNS results onto discrete mode $S$ and comparison with the theoretical prediction using the MMS; $f=44.76 \mathrm{kHz}$.

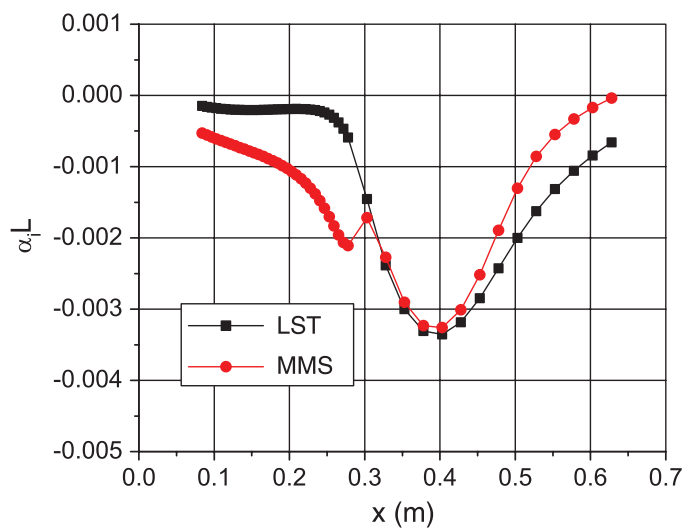

Fig. $15 \operatorname{Im}\left(\alpha_{i}\right)$ versus $x$ at frequency $f=104.44 \mathrm{kHz}$.

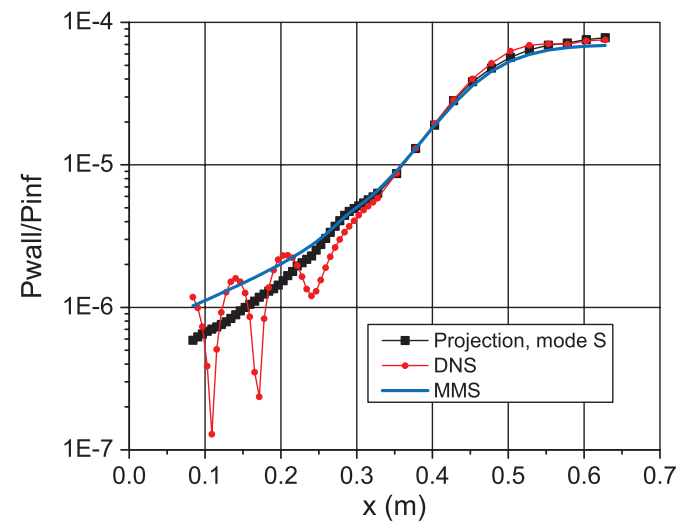

Fig. 16 Projection of the DNS results onto discrete mode $S$ and comparison with the theoretical prediction using the MMS; $f=104.44 \mathrm{kHz}$.

theoretical prediction when the nonparallel flow effects are included. Figures 15 and 16 show similar results corresponding to frequency $f=104.44 \mathrm{kHz}$. Figures 17 and 18 demonstrate the results at frequency $f=164.12 \mathrm{kHz}$. It is interesting that the nonparallel flow effect on $\alpha_{i} L$ has a different character at low and high local Reynolds numbers (see Figs. 15 and 17). Using Mack's terminology [33], mode $S$ is associated with the first Mack's mode and the second Mack's mode at low and high local Reynolds numbers, respectively (see clarification of the terminology in [34]). The nonparallel flow effects destabilize the first mode and stabilize the second mode. The same observation was reported by Chang and Malik [35]. Appearance of the strong hump in $\alpha_{i} L$ at low Reynolds numbers, as

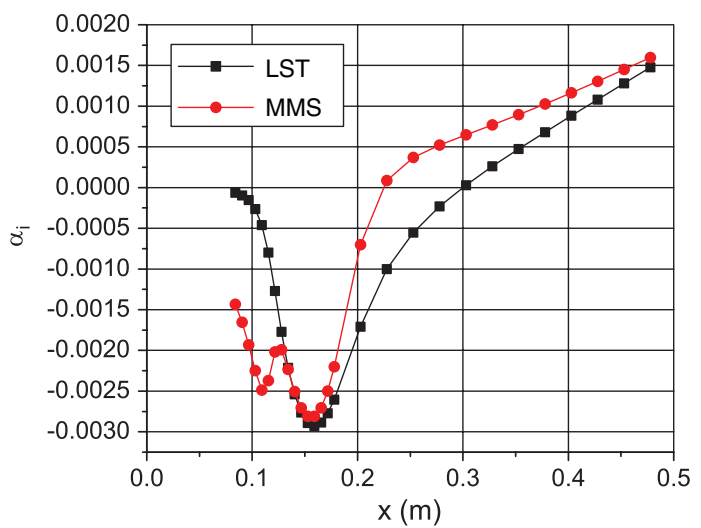

Fig. $17 \operatorname{Im}\left(\alpha_{i}\right)$ versus $x$ at frequency $f=164.12 \mathrm{kHz}$.

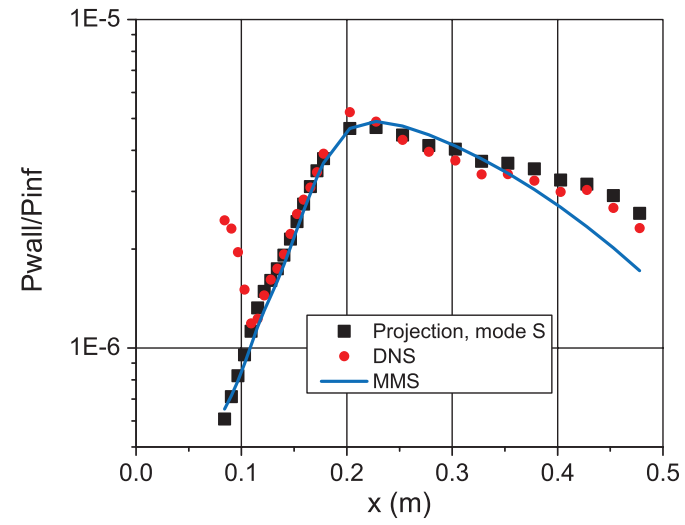

Fig. 18 Projection of the DNS results onto discrete mode $S$ and comparison with the theoretical prediction using the MMS; $f=164.12 \mathrm{kHz}$. 
seen in Figs. 15 and 17, is attributed to the transformation of the first mode into the second one [35].

\section{Conclusions}

The presented results illustrate how the multimode decomposition technique may serve as a tool for gaining insight into the flow dynamics in the presence of perturbations belonging to different modes. In the past, one could only compare DNS results with theoretical prediction for the unstable mode far downstream from an actuator, where the unstable mode dominates the total signal. Using the biorthogonal eigenfunction system, one can compare DNS results with theoretical predictions for the unstable and stable modes in the vicinity of the actuator as well.

In [4] and the present work, it has been found that the multimode decomposition requires a more elaborate analysis within the point of synchronism of mode $F$ with the continuous spectrum. Therefore, an extension of the theoretical model of [17] to the case of continuous spectrum is required.

Analysis of the growth rates using the MMS revealed that they have a nonmonotonic character in the region where the first Mack's mode is transformed into the second one (the terminology regarding Mack's modes is clarified in [34]). This observation is in agreement with studies in [35]. The nonparallel flow effects in the boundary layer over an adiabatic wall destabilize the first mode and stabilize the second one (see Figs. 15-17).

\section{Appendix A: Comparisons}

See Figs. A1-A6 for comparisons of the DNS mean velocity and temperature profiles with the self-similar solution.

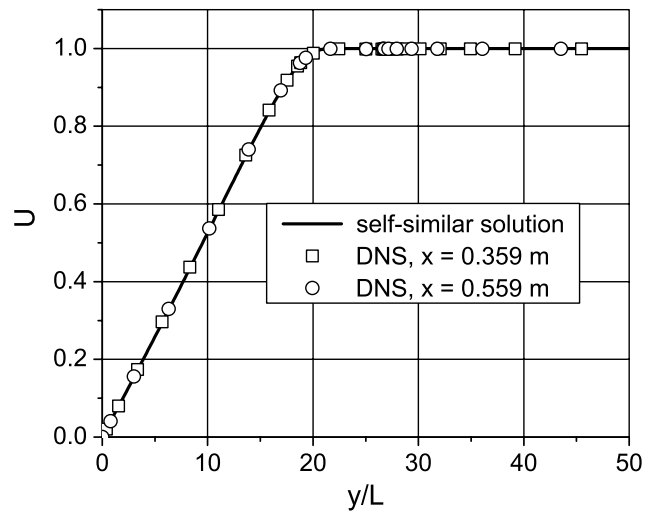

Fig. A1 Self-similar solution (solid line) and DNS results (symbols) for the mean velocity profile $U(y)$.

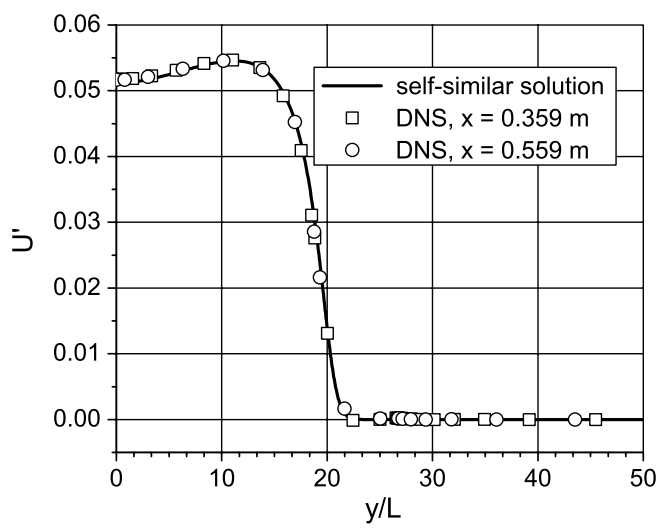

Fig. A2 Derivative $\mathrm{d} U / \mathrm{d} y$ : self-similar solution (solid line) and DNS (symbols)

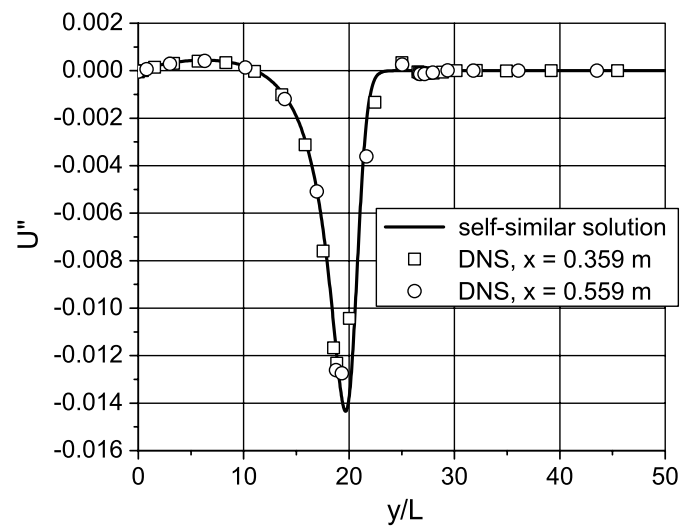

Fig. A3 Derivative $\mathrm{d}^{2} U / \mathrm{d} y^{2}$ : self-similar solution (solid line) and DNS (symbols).

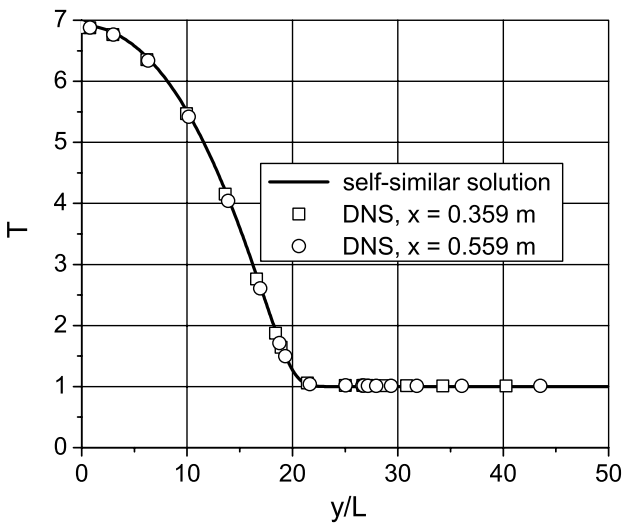

Fig. A4 Mean temperature profile $T(y)$ : self-similar solution (solid line) and DNS (symbols).

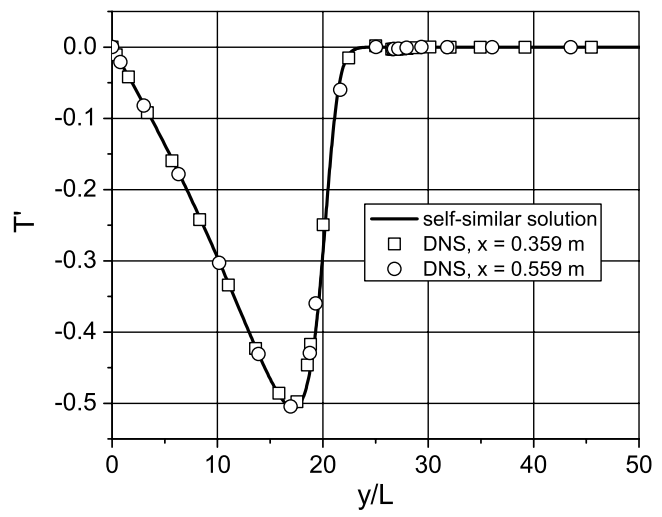

Fig. A5 Derivative dT/dy: self-similar solution (solid line) and DNS (symbols).

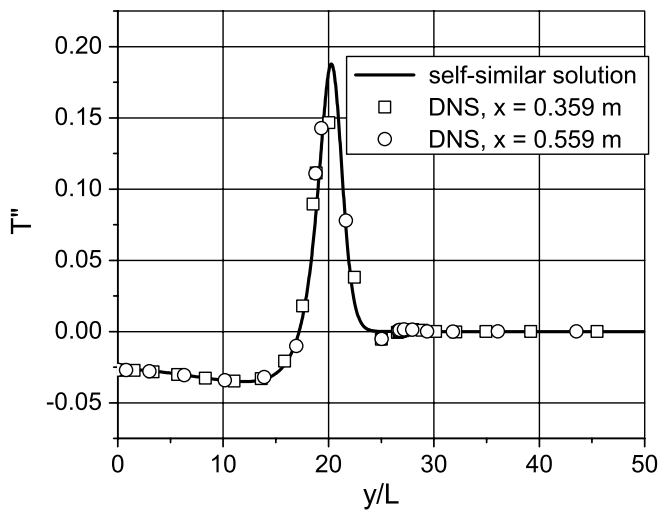

Fig. A6 Derivative $\mathrm{d}^{2} T / \mathrm{d} y^{2}$ : self-similar solution (solid line) and DNS (symbols). 


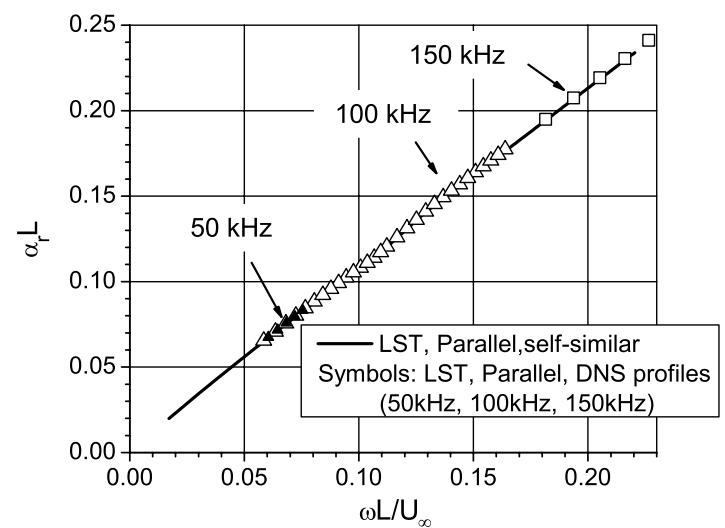

Fig. B1 Comparison of $\alpha_{r}$ obtained using DNS and self-similar velocity and temperature profiles: self-similar solution (solid line) and DNS profiles (symbols).

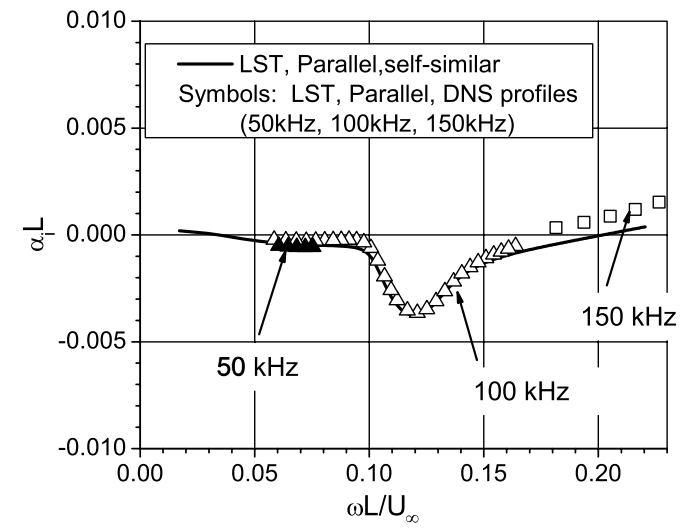

Fig. B2 Comparison of $\alpha_{i}$ obtained using DNS and self-similar velocity and temperature profiles: self-similar solution (solid line) and DNS profiles (symbols).

\section{Appendix B: Comparisons of Eigenvalues}

See Figs. B1 and B2 for comparisons of eigenvalues $\alpha=\alpha_{r}+i \alpha_{i}$ obtained using $\mathrm{DNS}$ and self-similar profiles.

\section{Acknowledgments}

This work was sponsored by the Air Force Office of Scientific Research (AFOSR) /National Center for Hypersonic Research in Laminar-Turbulent Transition and by the Air Force Office of Scientific Research, U.S. Air Force, under grants FA9550-08-10322 (A.T.) and FA9550-07-1-0414 (X.Z. and X.W.), monitored by J. D. Schmisseur. The views and conclusions contained herein are those of the authors and should not be interpreted as necessarily representing the official polices or endorsements, either expressed or implied, of the AFOSR or the U. S. Government.

\section{References}

[1] Tumin, A., "Multimode Decomposition of Spatially Growing Perturbations in a Two-Dimensional Boundary Layer," Physics of Fluids, Vol. 15, No. 9, 2003, pp. 2525-2540. doi: $10.1063 / 1.1597453$

[2] Gaydos, P., and Tumin, A., "Multimode Decomposition in Compressible Boundary Layers," AIAA Journal, Vol. 42, No. 6, 2004, pp. $1115-1121$. doi: $10.2514 / 1.2289$

[3] Tumin, A., "Three-Dimensional Spatial Normal Modes in Compressible Boundary Layers," Journal of Fluid Mechanics, Vol. 586, 2007, pp. 295-322. doi:10.1017/S002211200700691X

[4] Tumin, A., Wang, X., and Zhong, X., "Direct Numerical Simulation and the Theory of Receptivity in a Hypersonic Boundary Layer," Physics of
Fluids, Vol. 19, No. 1, 2007, Paper 014101. doi:10.1063/1.2409731

[5] Bouthier, M., "Stabilité Lineairé des Écoulements Presque Paralléles," Journal de Mecanique, Vol. 11, No. 4, 1972, pp. 599-621.

[6] Gaster, M., "On the Effects of Boundary Layer Growth on Flow Stability," Journal of Fluid Mechanics, Vol. 66, No. 3, 1974, pp. 465480. doi:10.1017/S0022112074000310

[7] Saric, W. S., and Nayfeh, A. H., "Nonparallel Stability of BoundaryLayer Flow," Physics of Fluids, Vol. 18, No. 8, 1975, pp. 945-950. doi:10.1063/1.861266

[8] Saric, W. S., and Nayfeh, A. H., "Nonparallel Stability of Boundary Layers with Pressure Gradients and Suction," AGARD CP 224, 1977.

[9] Padhaye, A. R., and Nayfeh, A. H., "Nonparallel Stability of ThreeDimensional Flows," AIAA Paper 1979-1281, 1979.

[10] Gaponov, S. A., "Influence of Nonparallel Flow on the Development of Disturbances in a Supersonic Boundary Layer," Fluid Dynamics, Vol. 15, No. 2, 1980, pp. 195-199. doi:10.1007/BF01342607

[11] Nayfeh, A. H., "Stability of Three-Dimensional Boundary Layers," AIAA Journal, Vol. 18, No. 4, 1980, pp. 406-416. doi: $10.2514 / 3.50773$

[12] El-Hady, N. M., "On the Stability of Three-Dimensional, Compressible Nonparallel Boundary Layers," AIAA Paper 1980-1374, 1980.

[13] Tumin, A. M., and Fedorov, A. V., "On The Weakly Nonparallel Flow Effect on Characteristics of Flow Stability," Uchenye Zapiski TSAGI, Vol. 13, No. 6, 1982, pp. 91-96 (in Russian).

[14] Herbert, T., and Bertolotti, F. B., "Stability Analysis of Nonparallel Boundary Layers," Bulletin of the American Physical Society, Vol. 32, 1987, pp. 2079.

[15] Bertolotti, F. P., "Linear and Nonlinear Stability of Boundary Layers with Streamwise Varying Properties," Ph.D. Thesis, Ohio State Univ., Columbus, OH, 1991.

[16] Herbert, T., "Parabolized Stability Equations," Annual Review of Fluid Mechanics, Vol. 29, 1997, pp. 245-283 doi:10.1146/annurev.fluid.29.1.245

[17] Fedorov, A. V., and Khokhlov, A. P., "Prehistory of Instability in a Hypersonic Boundary Layer," Theoretical and Computational Fluid Dynamics, Vol. 14, No. 6, 2001, pp. 359-375. doi: $10.1007 / \mathrm{s} 001620100038$

[18] Tumin, A., "Nonparallel Flow Effects on Roughness-Induced Perturbations in Boundary Layers," Journal of Spacecraft and Rockets, Vol. 45, No. 6, 2008, pp. 1176-1184. doi: $10.2514 / 1.37136$

[19] White, E. B., "Transient Growth of Stationary Disturbances in a Flat Plate Boundary Layer," Physics of Fluids, Vol. 14, No. 12, 2002, pp. $4429-4439$ doi: $10.1063 / 1.1521124$

[20] White, E. B., and Ergin, F. E., "Receptivity and Transient Growth of Roughness-Induced Disturbances," AIAA Paper 2003-4243, 2003.

[21] Fransson, J. H. M., Brandt, L., Talamelli, A., and Cossu, C., "Experimental and Theoretical Investigation of the Non-Modal Growth of Steady Streaks in a Flat Plate Boundary Layers," Physics of Fluids, Vol. 16, No. 10, 2004, pp. 3627-3638. doi:10.1063/1.1773493

[22] White, E. B., Rice, J. M., and Ergin, F. G., "Receptivity of Stationary Transient Disturbances to Surface Roughness," Physics of Fluids, Vol. 17, No. 6, 2005, Paper 064109. doi:10.1063/1.1938217

[23] Denissen, N.A., and White,E. B., "Roughness Induced Bypass Transition Revisited," AIAA Journal, Vol. 46, No. 7, 2008, pp. 1874-1877. doi:10.2514/1.35304

[24] Fischer, P., and Choudhari, M., "Numerical Simulation of RoughnessInduced Transient Growth in a Laminar Boundary Layer," AIAA Paper 2004-2539, 2004.

[25] Rizzetta, D. P., and Visbal, M. R., "Direct Numerical Simulations of Flow Past an Array of Distributed Roughness Elements," AIAA Journal, Vol. 45, No. 8, 2007, pp. 1967-1976. doi: $10.2514 / 1.25916$

[26] Stephani, K. A., Albright, J., Doolittle, C., Jackson, M., and Goldstein, D., "DNS Study of Transient Disturbance Growth and Bypass Transition," AIAA Paper 2009-0585, 2009.

[27] Denissen, N. A., and White, E. B., "Continuous Spectrum Analysis of Roughness-Induced Transient Growth," Physics of Fluids, Vol. 21, No. 11, 2009, Paper 114105. doi: $10.1063 / 1.3264090$

[28] Zhong, X., "High-Order Finite-Difference Schemes for Numerical Simulation of Hypersonic Boundary-Layer Transition," Journal of 
Computational Physics, Vol. 144, No. 2, 1998, pp. 662-709. doi:10.1006/jcph.1998.6010

[29] Maslov, A. A., Shiplyuk, A. N., Sidorenko, A. A., and Arnal, D., "Leading-Edge Receptivity of a Hypersonic Boundary Layer on a Flat Plate," Journal of Fluid Mechanics, Vol. 426, 2001, pp. 73-94. doi:10.1017/S0022112000002147

[30] Wang, X., and Zhong, X., "Effect of Wall Perturbations on the Receptivity of a Hypersonic Boundary Layer," Physics of Fluids, Vol. 21, No. 4, 2009, pp. 044101 doi: $10.1063 / 1.3103880$

[31] Friedman, B., Principles and Techniques of Applied Mathematics, Dover, New York, 1990.

[32] Malik, M. R., Lin, R. S., and Sengupta, R., "Computation of Hypersonic
Boundary-Layer Response to External Disturbances," AIAA Paper 1999-0411, 1999.

[33] Mack, L. M., "Boundary Layer Stability Theory," Jet Propulsion Lab., California Inst. of Technology Rept. 900-277, Pasadena, CA, 1969.

[34] Fedorov, A., and Tumin, A., "Branching of Discrete Modes in HighSpeed Boundary Layers and Terminology Issues," AIAA Paper 20105003, 2010.

[35] Chang, C.-L., and Malik, M. R., "Non-Parallel Stability of Compressible Boundary Layers," AIAA Paper 1993-2912, 1993.

F. Ladeinde Associate Editor 